\title{
TECNOLOGÍA CERÁMICA EN LA LOCALIDAD ARQUEOLÓGICA SAN PABLO (PENÍNSULA VALDÉS, PROVINCIA DEL CHUBUT. ARGENTINA)
}

\section{POTTERY TECHNOLOGY IN SAN PABLO ARCHAEOLOGICAL LOCALITY (PENÍNSUlA VALDÉS, CHUBUT PROVINCE. ARGENTINA)}

\author{
Verónica Schuster \\ IDEAus. CENPAT - CONICET. Boulevar A. Brown 2915 (CP 9120). Puerto Madryn, Chubut. \\ Email: schuster@cenpat-conicet.gob.ar
}

Presentado: $24 / 10 / 18$

Aceptado: 16/08/2019

\section{Resumen}

El presente trabajo aporta nueva información a los estudios ya emprendidos en la cerámica arqueológica de la costa noreste del Chubut, cuyo objetivo general es caracterizar la variabilidad, comprender el rol y estimar la antigüedad de esta tecnología entre las sociedades cazadorasrecolectoras que la implementaron. En esta oportunidad se presentan los resultados obtenidos del estudio tecno-morfológico y petrográfico efectuado al material cerámico recuperado en la localidad arqueológica San Pablo en la Península de Valdés. El buen estado de preservación, los contextos particulares de algunos hallazgos, la variabilidad morfológica de ciertos ejemplares, así como la presencia de una pieza completa, permiten avanzar en la interpretación y discusión de la manufactura y circulación de esta tecnología en la región hacia circa los 900 años AP.

Palabras clave: cerámica arqueológica, cazadores-recolectores, costa de Patagonia, Península Valdés.

\begin{abstract}
The present work brings new information to the studies already undertaken in the archaeological pottery of the northeast coast of Chubut. Its general objective is to characterize the variability, understand the role and estimate the chronology of this technology among the huntergatherer groups that implemented it. The results obtained from the techno-morphological and petrographic study carried out on the ceramic material recovered at the archaeological site of San Pablo in the Valdés Peninsula are presented on this occasion. The grade state of preservation, the particular contexts of some findings, morphological variability of certain specimens as well as the presence of a complete piece, allow progress in the interpretation and discussion of the manufacture and circulation of this technology in the region to circa 900 years ago.
\end{abstract}

Keywords: archaeology pottery, hunter-gatherers, Patagonian coast, Peninsula Valdés. 


\section{Introducción}

El área de estudio que se presenta comprende la costa nordeste de la provincia del Chubut. Específicamente, la localidad arqueológica en la que se centra este trabajo -San Pablo- se emplaza sobre el extremo SO de la Península Valdés y se corresponde con una amplia bajada litoral (Figura 1 y 2). Los rasgos geomorfológicos asociados con la paleodinámica costera sugieren que esta bajada litoral habría sido utilizada luego de la máxima transgresión del Holoceno medio.

Por su parte, los estudios arqueológicos emprendidos durante los años 2012 al 2014 permitieron detectar una tendencia de la ocupación humana relacionada con el gradiente atitudinal: a menor altura sobre el nivel del mar, mayor densidad y diversidad funcional de sitios arqueológicos (Gómez Otero et al. 2013; Gómez Otero et al. 2017a). De los 11 sitios registrados, seis de ellos presentan cerámica y se ubican en las cotas más bajas (entre 5 y $3 \mathrm{msnm}$ ), cerca de la línea de costa actual (menos de 850m aproximadamente). El material cerámico se presenta fragmentario, aunque muestra distintos grados de preservación, registrándose hasta el momento más de 200 tiestos y una vasija completa. También se dispone de dataciones de algunos contextos cerámicos que indican una antigüedad aproximada de entre 800 y 900 años AP para esta tecnología en el área (Tabla 1). Sin embargo, fechados obtenidos de restos óseos humanos permiten sustentar que este espacio costero fue ocupado por lo menos desde hace 3000 años AP (Gómez Otero et al. 2017a).

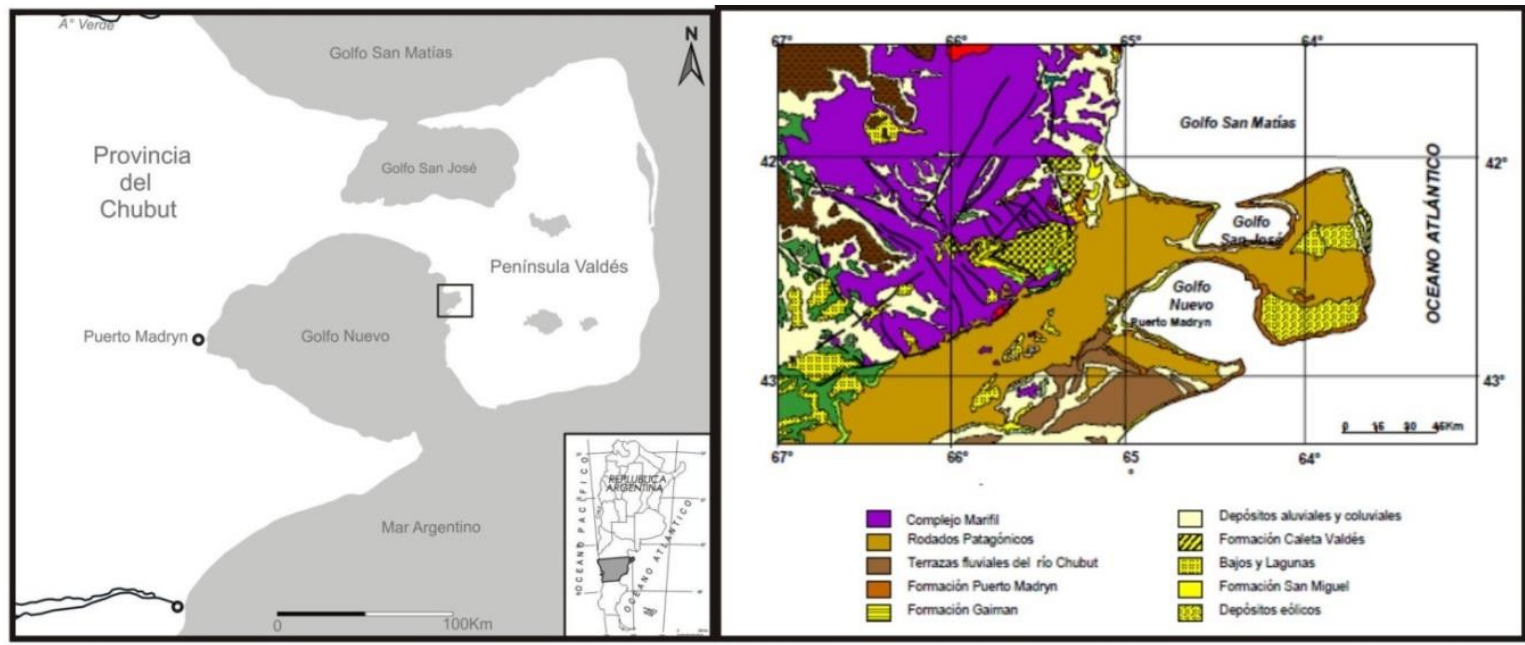

Figura 1. a) Área de estudio y ubicación de la bajada Colombo donde se emplaza la localidad arqueológica San Pablo; b) geología regional simplificada (tomado y modificado de Haller et al. 2005).

El presente trabajo tiene como objetivo presentar una nueva localidad arqueológica con registro cerámico para la costa nordeste del Chubut y reseñar los resultados del estudio tecno-morfológico y petrográfico obtenido de dicho material. Estos datos permiten 
avanzar en el objetivo más general de la investigación en curso tendiente a la caracterización, interpretación y discusión de la manufactura y circulación de esta tecnología en la región.

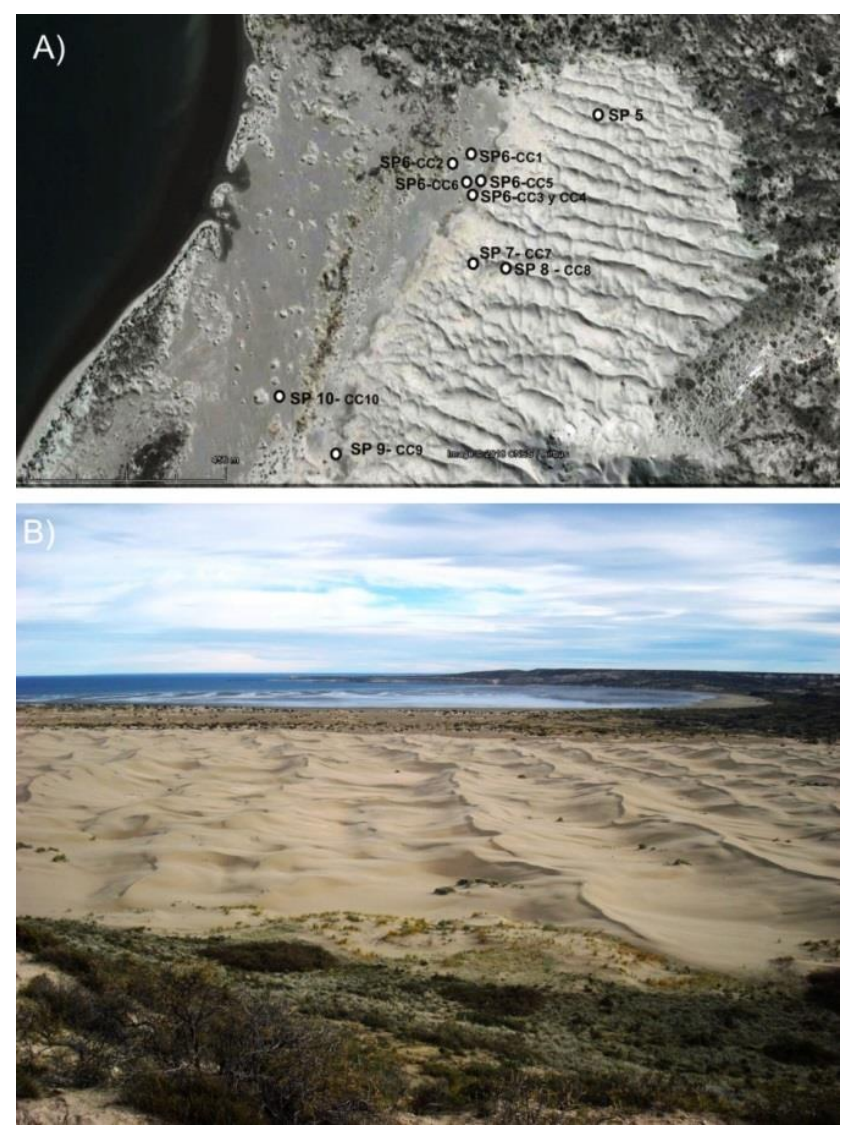

Figura 2. a) Sitios con registro cerámico de la localidad arqueológica San Pablo; b) fotografía de la franja de médanos de la bajada Colombo tomada desde el E.

\begin{tabular}{|c|c|c|c|c|c|}
\hline Sitio & Contexto & $\begin{array}{c}\text { Código } \\
\text { Laboratorio }\end{array}$ & $\begin{array}{c}\text { Edad C14 AP } \\
\text { Convencional }\end{array}$ & $\begin{array}{c}\text { Edad } \\
\text { Calibrada 1 } \sigma\end{array}$ & $\begin{array}{c}\text { Material } \\
\text { Datado }\end{array}$ \\
\hline SP 6 & Muestreo 1 (basural) & LP-2807 & $400 \pm 50$ & $\begin{array}{c}1457-1511 \\
\text { DC }\end{array}$ & Carbón \\
\hline SP 7 & $\begin{array}{c}\text { Concentración de } \\
\text { carbones }\end{array}$ & LP- 2802 & $880 \pm 60$ & $1159-1266$ & Carbón \\
& DC & \\
\hline SP 7 & $\begin{array}{c}\text { Paquete de guanaco } \\
N^{\circ} 1\end{array}$ & LP- 2894 & $980 \pm 70$ & $1030-1162$ & Óseo \\
& & & DC & guanaco \\
\hline
\end{tabular}

Tabla 1. Fechados radiocarbónicos convencionales y calibrados de contextos con registro cerámico de la localidad arqueológica San Pablo (Gómez Otero et al. 2017a). La calibración en años calendáricos es de un sigma (68\% de probabilidad) según el programa CALIB 6.0.1, en conjunción con Stuiver y Reimer (1993). 


\section{Localidad Arqueológica San Pablo}

Aspectos geomorfológicos y geología

Esta localidad arqueológica se corresponde con una amplia bajada litoral denominada Bajada Colombo- de $5 \mathrm{~km}$ de longitud y $1.5 \mathrm{~km}$ de ancho aproximadamente.

La geomorfología de la Península presenta playas con suaves bajadas y escasa pendiente que gradualmente alcanzan el mar, y mesetas de no más de $100 \mathrm{msnm}$ que culmina en acantilados abruptos. Otro rasgo característico son los médanos litorales (Súnico 1996) que se presentan de manera discontinua, alcanzando alturas muy variables. En el sector sur de Península Valdés conforman un extenso campo de médanos fijos y/o activos que se desarrolla en sentido este-oeste (Figura 2). Estas dunas están modeladas por procesos deflacionarios que desarrollan numerosas hoyadas de deflación, y que en situaciones extremas, suelen dejar expuesto el sustrato del terciario. Precisamente, estos sectores que ofrecen ambientes amplios, poco vegetados y con reparo del viento, es donde se registran frecuentemente los sitios y los hallazgos arqueológicos en el área de estudio (Gómez Otero 2007).

La geología está representada principalmente por rocas sedimentarias marinas de edad terciaria y depósitos del Cuaternario (Haller 1994). Las sedimentitas corresponden a las Formaciones Gaiman y Puerto Madryn del Terciario Superior. La primera está constituida por areniscas limosas, limolitas, tobas con abundante material vítreo y fangolitas cineríticas (sedimentitas limo-arcillosas con contenido de cenizas volcánicas), representando un ambiente marino somero (Haller 1994). La Formación Puerto Madryn constituida por areniscas y pelitas con abundante contenido fosilífero (Scasso y Del Río 1987)- representaría un ambiente marino somero con sus términos superiores transicionales a ambientes continentales.

Por su parte, el Cuaternario se halla representado por los Rodados Patagónicos, la Formación San Miguel -representada por depósitos de playa antiguos, compuestos por gravas arenosas y numerosos restos de conchillas- y las arenas y sedimentos finos de bajos y lagunas. Finalmente, la Formación Caleta Valdés corresponde a los antiguos cordones litorales compuestos por gravas medianas a gruesas bien redondeadas de edad pleistocena. Estos depósitos se localizan en el extremo oriental de la Península Valdés, específicamente, en toda la margen occidental de la Caleta Valdés (Haller et al. 2001).

En el área costera, la erosión de las unidades geológicas aflorantes -que se presentan como depósitos indiferenciados de aluvio, coluvio y/o de bajos y lagunas- son materiales finos como limos o arcillas factibles de ser empleados para la producción cerámica. 
Registro Arqueológico:

San Pablo se caracteriza por la presencia de sitios en superficie, pudiéndose identificar removilización de materiales de distintos eventos de ocupación (palimpsesto) debido a los agentes naturales que imperan en este tipo de ambiente, fundamentalmente, la acción eólica. Igualmente, no hay que descartar la acción hídrica, la dinámica marina, el tránsito y el pisoteo de los animales y el factor antrópico (Gómez Otero et al. 2017a).

Para esta área se contaba con la información previa de un único sitio -San Pablo 1 (SP1)- localizado en una hoyada entre médanos del que se recuperaron puntas de proyectil de basalto en casi todos los estadios de manufactura. De acuerdo a estos rasgos, se caracterizó al sitio como un taller de confección de piezas bifaciales (Gómez Otero et al. 1999). En las tareas de campo de los últimos años se reconocieron 10 nuevos sitios arqueológicos (Gómez Otero et al. 2013, Gómez Otero et al. 2017a). A continuación, se describen brevemente los que presentan registro cerámico, y cuyos materiales son analizados en este trabajo (Figura 2).

San Pablo 5 (SP5)

Es una pequeña concentración de superficie que presenta materiales muy dispersos (principalmente restos faunísticos) en una superficie de 5.80 por 8 metros, y a casi $1 \mathrm{~km}$ de la línea de costa actual. En un muestreo de $2 \times 2$ metros se identificaron huesos de guanaco (principalmente fragmentos de vértebras), una falange de lobo marino de un pelo (Otaria flavensis), placas de armadillo, algunas astillas de huesos quemados sin identificar y valvas muy pequeñas disgregadas. También se recuperaron instrumentos líticos: una punta pedunculada de basalto y dos raspadores de calcedonia. Entre los pocos desechos de talla, se recogieron dos lascas de calcedonia. Se destaca además el hallazgo de una cuenta o chaquira muy pequeña realizada sobre una valva de Fissurella y un fragmento cerámico muy pequeño. Se estimó que este espacio fue utilizado para el procesamiento y consumo de distintos recursos terrestres y marinos, y donde también se habrían desarrollado actividades de producción de talla lítica (Gómez Otero et al. 2017a).

San Pablo 6 (SP6)

Se localiza en una laguna costera entre dos cordones de médanos paralelos a la costa, a 3 msnm y a 500 metros de la línea de costa actual (Figura 3). Se presenta como un montículo o una acumulación artificial de forma semilunar de $15 \mathrm{~cm}$ de alto aproximadamente. En la superficie del mismo -que abarca $15 \mathrm{~m}$ de largo por $5 \mathrm{~m}$ de anchose registraron fragmentos de huesos quemados y meteorizados, tiestos cerámicos muy pequeños y diversos artefactos líticos fracturados. 
El análisis efectuado a los dos mil especímenes óseos recuperados de un muestreo de 2x2m, permitió constatar la explotación de una amplia variedad de vertebrados terrestres y marinos de distintos tamaños. Se determinó un número mínimo de dos individuos de guanaco y cuatro pinnípedos, identificándose lobo marino de un pelo y lobo marino de dos pelos (Arctocephalus australis), esta última especie no se encuentra disponible en Península de Valdés en la actualidad. También, se identificaron cormoranes (Phalacrocoraxs pp.), pingüino de Magallanes (Spheniscus magellanicus) y pequeños mamíferos como piches (Zaedyus pichiy) y peludos (Chaetophractus villosus) (Svodoba 2015). Otras especies halladas corresponden a un atún y a un delfín común (Delphinus delphis) o delfín oscuro (Lagenorhynchus obscurus) (Atila Gosztonyi, Com. Pers., 2013) y restos óseos actuales de ballena.

El sitio SP6 representaría un área de descarte o basural de uno o más sitios domésticos extensos que habrían funcionado como bases residenciales, con una antigüedad de por lo

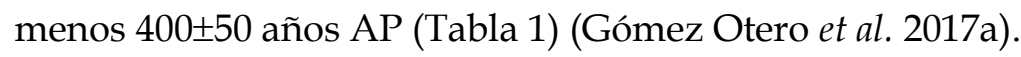

SP6 - Concentración Cerámica 1 (CC1). Se emplaza a 50 metros hacia el este de SP6, y se compone de 14 fragmentos que se presentaban sobre un sedimento semi-consolidado con espículas carbonosas que se encontraba disectado por la acción hídrica. Se realizó una recolección de superficie de todos los tiestos (Figura 3).

SP6 - Concentración Cerámica 2 (CC2). Se compone de 57 fragmentos que se recuperaron en el muestreo realizado sobre el sitio SP6 y en inmediaciones de este contexto, y que posiblemente correspondan a materiales trasportados o removilizados por agentes naturales y/o antrópicos.

SP6 - Concentración Cerámica 3 (CC3). Se emplaza a 20 metros al este de SP6 (Figura 3). Se realizó la recolección de superficie de 17 fragmentos que se encontraron junto a otros artefactos líticos grandes y pesados (un molino plano y varios percutores).

SP6 - Concentración Cerámica 4 (CC4). Se ubica a menos de 10 metros de la CC3 y está compuesta por 31 fragmentos asociados a un molino plano de arenisca, un percutor, cáscaras de huevo de choique y algunos huesos pequeños quemados sin identificar. Los tiestos fueron recuperados a partir de una excavación arqueológica (Gómez Otero et al. 2017a) (Figura 3).

SP6 - Concentración Cerámica 5 (CC5). Se ubica a menos de 10 metros de la CC3 y está compuesta por 12 fragmentos que corresponderían a dos piezas diferentes. Los tiestos recuperados en superficie- se encontraron junto a cascaras de huevo de choique y un probable instrumento de hueso de ave. 
SP6 - Concentración Cerámica 6 (CC6). Corresponde a 21 tiestos que se hallaron en superficie, sin ningún otro resto arqueológico asociado.

San Pablo 7 (SP7)

Se trata de un conjunto de cinco concentraciones o paquetes de huesos de guanaco dispuestos artificialmente en una hoyada entre médanos hacia el este de la laguna costera, a $4 \mathrm{msnm}$ y a 630 metros de la línea de costa actual. La particularidad de estas concentraciones es que presentan una disposición artificial de los restos (Figura 3). Dos de estos conjuntos fueron excavados ( $\mathrm{N}^{\mathrm{o}} 1$ y 3 ) registrándose la disposición paralela -y con dirección predominante E-O- de seis hileras de segmentos de la columna vertebral de cuatro guanacos (correspondiendo su mayoría a adultos), de las que además, habían sido removidas las espinas transversales en gran parte de ellas. Asimismo, se registraron relictos de fogones -uno de ellos datado (Tabla 1), restos arqueofaunísticos -almeja blanca, buchinos, placas de peludo, huesos de pingüino- y materiales líticos: un raspador frontal de calcedonia, un limbo de calcedonia, un perforador de cuarcita, dos sobadores de riolita, varios percutores fragmentados, una bola de boleadora partida y algunos desechos de talla (Gómez Otero et al. 2017a).

La disposición particular de los huesos -en discretas concentraciones y con un acondicionamiento particular de los restos óseos- no puede interpretarse únicamente como producto de su trozamiento y/o consumo, por lo que se infiere algún tipo de práctica simbólica o ritual cuyo registro en la región patagónica es hasta el momento inédita.

SP7 - Concentración Cerámica 7 (CC7).Corresponde a 63 fragmentos que pertenecen a una única pieza (Figura 3 y 4) que se encontraban a siete metros de las concentraciones de guanacos. Además, en la misma hoyada fueron recuperados dos tiestos muy pequeños sin asociación contextual.

San Pablo 8 (SP8)

Se emplaza en una hoyada en un campo de dunas, distante unos 20 metros al este de SP7, a 4 msnm y a $600 \mathrm{~m}$ de la línea de costa. Se realizó una recolección asistemática de materiales en superficie en la que se identificaron restos faunísticos de guanacos y pinnípedos, fragmentos cerámicos y materiales líticos: desechos de talla de ignimbrita, obsidiana y calcedonia, un raspador microlítico de calcedonia y un fragmentode roca sedimentaria con incisiones que podría corresponderse con una placa grabada. Se interpretó que en este sitio se trozaron y consumieron guanacos y pinnípedos (Gómez Otero et al. 2017a). 

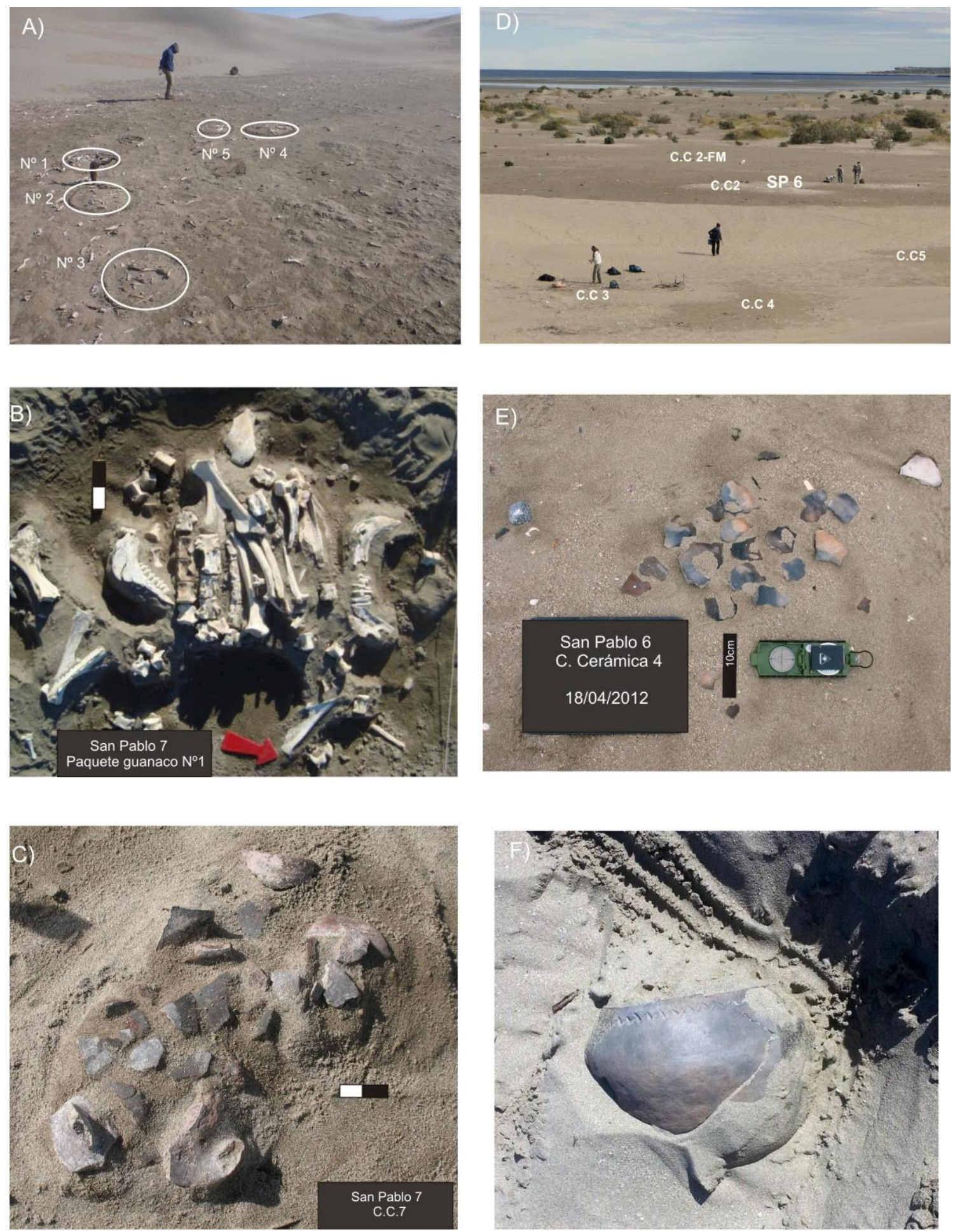

Figura 3:a) SP7: paquetes de restos óseos de guanaco; b) detalle de la excavación del paquete óseoN ${ }^{\mathrm{o}} 1$; c) CC7 asociada a los contextos óseos de guanacos-SP7;d) SP6 y concentraciones cerámicas asociadas: $\mathrm{CC} 2, \mathrm{CC} 3, \mathrm{CC} 4$ y CC5;e) detalle de la excavación de la CC4; f) pieza cerámica completa in situ. 
SP8 - Concentración Cerámica 8 (CC8).Compuesta por nueve fragmentos sin otra asociación material ni contextual.

San Pablo 9 (SP9)

Es un entierro humano primario individual emplazado sobre la laguna costera, a 3 msnm y a $600 \mathrm{~m}$ de la costa, que se encontraba en muy buen estado de preservación. El individuo -masculino, de entre 20 y 35 años de edad- fue sepultado sobre su lado izquierdo y presentaba un aplanamiento en la parte posterior del cráneo (deformación tabular erecta). Se recuperó una cuenta pequeña de valva de lapa (Fissurella sp.) y una pequeña punta de proyectil fracturada asociada, además de pigmento mineral rojo sobre el cráneo y el tórax (Gómez Otero et al. 2017a).

SP9 - Concentración Cerámica 9 (CC9): Sobre una duna de baja altura, y a 20 metros del enterratorio humano se recuperaron 20 fragmentos en superficie sin otro tipo de material arqueológico asociado.

San Pablo 10 (SP10)

Es una pequeña concentración de artefactos líticos (en análisis) y fragmentos cerámicos sobre un sedimento húmedo de la laguna costera, a 4 m.s.n.m. y a 600 metros del mar (Gómez Otero et al. 2017a).

SP10 - Concentración Cerámica 10 (CC10): Compuesta por 19 tiestossin ningún otro resto arqueológico asociado.

\section{Materiales y Métodos}

El total del material analizado asciende a 302 fragmentos y a una pieza completa que fue recuperada por un poblador local. Para este conjunto se emprendieron análisis macro y microscópicos que se mencionan brevemente a continuación.

1) Estudios macroscópicos: En la totalidad de la muestra se estimaron las características generales (espesor, tamaño, peso, etc.), los aspectos relativos a su preservación (redondeamiento, erosión, etc.) y las variables tecnológicas como técnicas de manufactura, acabado de superficie y decoración. Además, se realizaron tareas de ensamblajes que permitieron reconstruir parcialmente algunas piezas. Se describieron también todos los segmentos diagnósticos reconocidos, y particularmente a partir de los bordes, se estimó el EVE o equivalente de vasijas estimadas (Orton et al. 1997). 
2) Estudios microscópicos (petrografía): Se realizó un muestreo de fragmentos para realizar cortes delgados con la finalidad de determinar la composición de las pastas y estimar la procedencia de la materia prima empleada. Para esta selección se tomaron en cuenta principalmente las piezas que pudieron ser parcialmente reconstruidas, los segmentos diagnósticos, los fragmentos decorados y/o los que presentaran pastas con inclusiones potencialmente no locales (mica), obteniéndose seis fragmentos para estudios petrográficos (Tabla 2). Para profundizar otros aspectos de los criterios teóricometodológicos empleados en los análisis macro y microscópicos se sugiere consultar alguno de los trabajos ya publicados: Schuster 2009, 2010, 2014, 2015, entre otros.

\begin{tabular}{|c|c|c|c|}
\hline Sitio & Concentración Cerámica & No $^{\text {Muestra Petrográfica }}$ & Segmento Diagnóstico \\
\hline SP 6 & 2 & 126 & Cuerpo-borde \\
& 3 & 125 & Cuerpo \\
& 4 & 128 & Indeterminado \\
& 9 & 129 & Cuerpo \\
\hline SP 7 & 1 & 124 & Borde \\
\hline SP 9 & 5 & 127 & Cuerpo-borde \\
\hline
\end{tabular}

Tabla 2: Muestras cerámicas seleccionadas para efectuar los estudios petrográficos.

\section{Resultados obtenidos}

Análisis Macroscópicos:

En la Tabla 3 se resumen los datos macroscópicos obtenidos. Las concentraciones cerámicas 2, 4 y 7 fueron las que mayor densidad de fragmentos presentaron: entre 40 y más de 70 tiestos (Tabla 3). Cabe destacar que el 17.54\% ( $\mathrm{N}=53)$ del material no ha sido considerado en este estudio debido a su pequeño tamaño (menor a $2 \mathrm{~cm}^{2}$ ). Respecto de la preservación del conjunto, además de los fragmentos pequeños, se detectaron tiestos rodados $(6.62 \%)$ y exfoliados $(46.35 \%)$.

Las tareas de remontaje permitieron obtener 19 unidades que contienen 88 tiestos (29.13\% del total de fragmentos). Se destaca que en la mayoría de las concentraciones se establecieron ensamblajes, y que en la CC4 se obtuvo el mayor número de fragmentos remontados debido a las fracturas frescas y a la buena preservación general de los tiestos. Por el contrario, en la CC5 no se establecieron remontajes a causa de la exfoliación de los mismos (Tabla 3).

Con relación a los segmentos diagnósticos, en primer orden se presentan los cuerpos, le continúan los bordes, y en menor medida, bases y asas (Tabla 3). Los tipos de bordes en su mayoría son rectos o levemente invertidos, con labios rectos o cóncavos. A partir de 
estos se pudo establecer el EVE, destacándose la CC2 y CC6 con dos piezas representadas respectivamente (Tabla 3). Asimismo, se estimó un diámetro de boca que varía entre los 10 y $22 \mathrm{~cm}$.

La única pieza completa de este conjunto corresponde a una olla globular de base cóncava que presenta $16 \mathrm{~cm}$ de diámetro. El borde es levemente invertido, con labio cóncavo. Las superficies están alisadas y presenta una decoración incisa en zig-zag que se ejecutó a1 cm del borde. Esta pieza fue trabajada a partir de la reconstrucción digital en 3D (Schuster y Quinto Sánchez 2019) obteniéndose datos del volumen -menor a 2 litros- y del peso del contendor vacío: $0.28 \mathrm{~kg}$. (Figura $4 \mathrm{c}$ ). Al igual que esta pieza, otras parcialmente reconstruidas muestran también formas levemente restringidas con diámetros de boca que varían entre 10 y 18 cm: SP6-CC3, pieza 1 y 2, SP6-CC5 (Figura 4). Por otra parte, las formas abiertas se encuentran representadas hasta el momento por solo dos casos -SP6, CC10, pieza 1 y 2 - y cuyos diámetros no superan los $12 \mathrm{~cm}$.
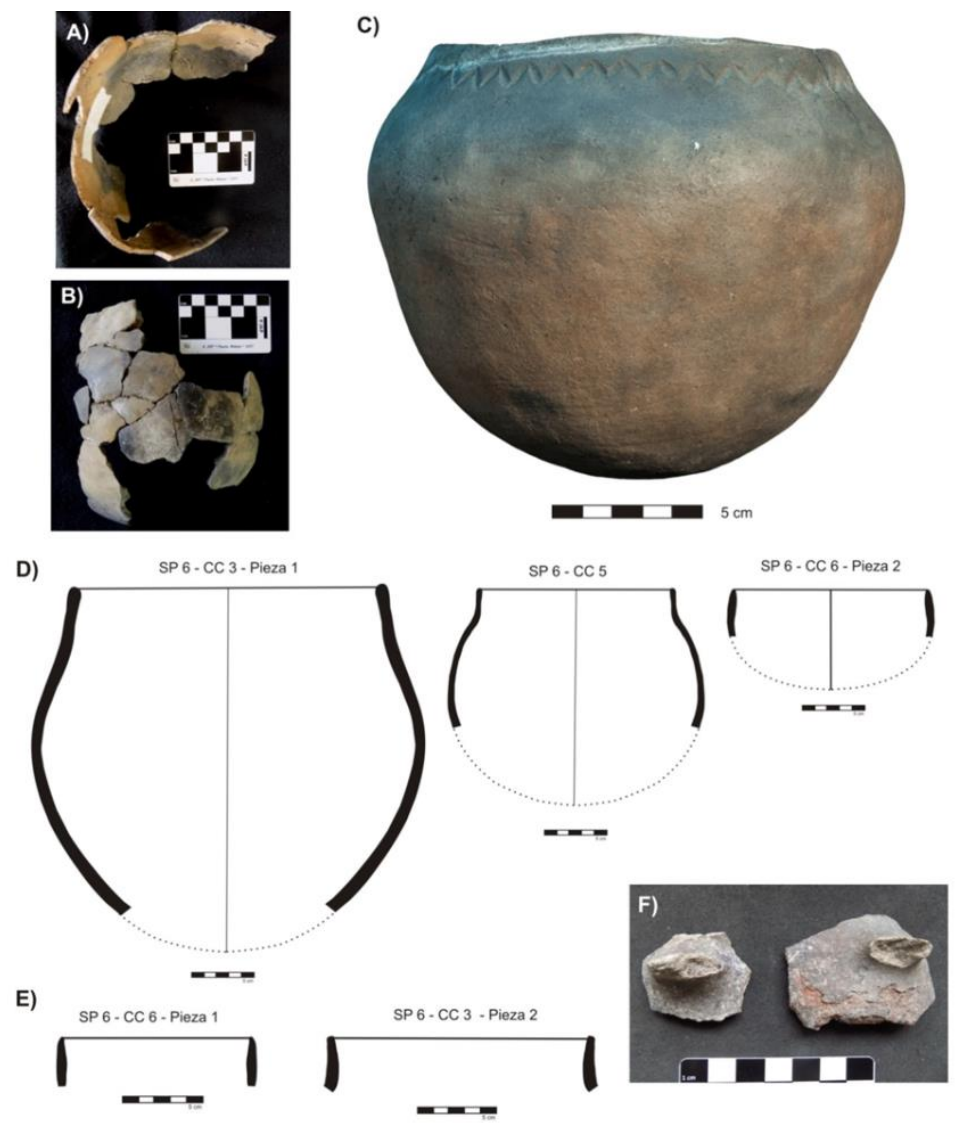

Figura 4. a y b) dos piezas parcialmente ensambladas de la CC4; c) la única pieza completa recuperada en la localidad;d) reconstrucciones de piezas remontadas en SP6; e) dibujos de bordes de SP6;f) asas de la pieza recuperada en SP7-CC7. 


\begin{tabular}{|c|c|c|c|c|c|c|c|c|c|}
\hline Sitios & $\begin{array}{c}\mathrm{N}^{\mathrm{O}} \\
\text { Tiestos }\end{array}$ & $\begin{array}{c}\text { EVE } \\
\text { Bordes }\end{array}$ & $\begin{array}{l}\text { Segmentos } \\
\text { Diagnósticos }\end{array}$ & $\begin{array}{l}\text { No Grupos } \\
\text { Macroscópicos }\end{array}$ & $\begin{array}{c}\text { Espesor } \\
\text { Promedio* }\end{array}$ & $\begin{array}{l}\text { Tratamiento de } \\
\text { Superficie }\end{array}$ & Decoración & Ensamblajes & Manufactura \\
\hline $\begin{array}{l}\text { SP6 } \\
\text { c.c.1 }\end{array}$ & 16 & - & - & 1 & 6.45 & Alisado int/ext & - & $\begin{array}{l}3 \text { unid. de } 7 \\
\text { fragm. }\end{array}$ & $?$ \\
\hline $\begin{array}{l}\text { SP6 } \\
\text { c.c. } 2\end{array}$ & 70 & 2 & Base & 1 & $\begin{array}{c}3.50 \\
(8.50 \text { base) }\end{array}$ & Alisado int/ext & Incisa & $\begin{array}{l}3 \text { unid. de } 10 \\
\text { fragm. }\end{array}$ & $?$ \\
\hline $\begin{array}{l}\text { SP6 } \\
\text { c.c. } 3\end{array}$ & 25 & 1 & Borde & 1 & 5.15 & Alisado int/ext & - & $\begin{array}{l}2 \text { unid. de } 13 \\
\text { fragm }\end{array}$ & Enrollam. \\
\hline $\begin{array}{l}\text { SP6 } \\
\text { c.c. } 4\end{array}$ & 41 & 1 & Borde & 2 & 4.47 y 2.64 & Alisado int/ext & - & $\begin{array}{l}3 \text { unid. de } 34 \\
\text { fragm. }\end{array}$ & Enrollam. \\
\hline $\begin{array}{l}\text { SP6 } \\
\text { c.c. } 9\end{array}$ & 12 & 1 & Borde & 1 & 6.00 & Alisado int/ext & - & $\begin{array}{l}2 \text { unid. de } 6 \\
\text { fragm. }\end{array}$ & $?$ \\
\hline $\begin{array}{l}\text { SP6 } \\
\text { c.c.10 }\end{array}$ & 21 & 2 & Borde & 2 & $\begin{array}{c}5.83 \mathrm{y} \\
6.48\end{array}$ & Alisado int/ext & - & $\begin{array}{l}3 \text { unid. de } 12 \\
\text { fragm. }\end{array}$ & $?$ \\
\hline $\begin{array}{l}\text { SP7 } \\
\text { c.c. } 7\end{array}$ & 74 & 1 & $\begin{array}{l}\text { Borde y } \\
\text { dos asas }\end{array}$ & 3 & 4.78 & $\begin{array}{l}\text { Pulido int/ext y } \\
\text { Alisado int/ext }\end{array}$ & - & $\begin{array}{l}2 \text { unid. de } 4 \\
\text { fragm. }\end{array}$ & Enrollam. \\
\hline $\begin{array}{l}\text { SP8 } \\
\text { c.c. } 8\end{array}$ & 12 & - & - & 2 & $\begin{array}{c}4.27 \mathrm{y} \\
4.45\end{array}$ & $\begin{array}{l}\text { Alisado int/ext. y } \\
\text { Alisado int/Pulido } \\
\text { ext }\end{array}$ & Engobe rojo & - & $?$ \\
\hline $\begin{array}{l}\text { SP9 } \\
\text { c.c. } 5\end{array}$ & 12 & 1 & Borde & 1 & 5.47 & Alisado int/ext & - & $\begin{array}{c}1 \text { unid.de } 2 \\
\text { fragm }\end{array}$ & $?$ \\
\hline $\begin{array}{l}\text { SP10 } \\
\text { c.c. } 6\end{array}$ & 19 & - & - & 1 & 3.50 & $\begin{array}{c}\text { S/ Ident. } \\
\text { (fragm exfoliados) }\end{array}$ & - & - & $?$ \\
\hline
\end{tabular}

Referencias: *en mm.

Tabla 3. Resultados de los estudios macroscópicos de la cerámica de la localidad San Pablo. Nota: Indet. (indeterminado), int. (interno), ext. (externo), unid (unidades), fragm. (fragmentos), enrollam. (enrollamiento). 
En cuanto a los acabados de superficie y decoración, la mayor parte del conjunto se caracteriza por el alisado interno y externo, destacándose un caso pulido, uno con engobe rojo y uno inciso (Tabla 3). Asimismo, la única pieza completa del conjunto presenta una decoración incisa en zig-zag (Figura 4).

Con relación a la técnica de manufactura, solo en tres casos se pudo distinguir macroscópicamente que la elaboración se habría llevado a cabo a partir del enrollamiento (Tabla 3).

Finalmente, a partir del registro y análisis de todas las variables o atributos que presentan los fragmentos-y siguiendo la propuesta general de Orton et al. (1997)- se identificaron 15 "familias de fragmentos" o grupos cerámicos macroscópicos (Tabla 3).

\section{Análisis Microscópicos:}

La muestra $N^{o} 124$-que corresponde a la cerámica recuperada en el contexto de restos óseos de guanacos- es la única estudiada microscópicamente que presenta mica como elemento mayoritario (Tabla 4 y Figura 5). De acuerdo a la geología local y los datos previos obtenidos en la tecnología cerámica de la zona, ésta podría corresponder a una pieza alóctona (Schuster 2009, 2014, 2019).Otro aspecto que refuerza esta hipótesis es la presencia de dos apéndices fracturados que se corresponderían con asas. Dichos atributos no son habituales en la cerámica de la Patagonia Argentina ni en la región en estudio (Schuster 2014, 2016, 2019), si bien sí lo son para muchos de los tipos cerámicos identificados en el centro-sur de Chile (Adán et al. 2005; Bullock 1973; Munita et al. 2011, entre otros autores).

La composición mineralógica de los restantes cortes delgados (Tabla 4) se corresponde con las características generales de los materiales cerámicos ya analizados en la región, y que habrían sido producidos localmente. Esto se establece por la presencia de cuarzo, feldespatos potásicos, plagioclasas, minerales opacos, fragmentos líticos de origen volcánico -si bien variando su representación en las muestras-, y finalmente, por anfíboles y piroxenos como elementos minoritarios (Figura 5). También, es distintivo en las pastas la presencia de vidrio volcánico y de mica en muy bajas proporciones, tal como se ha registrado en los materiales de la localidad San Pablo (Tabla 4). 

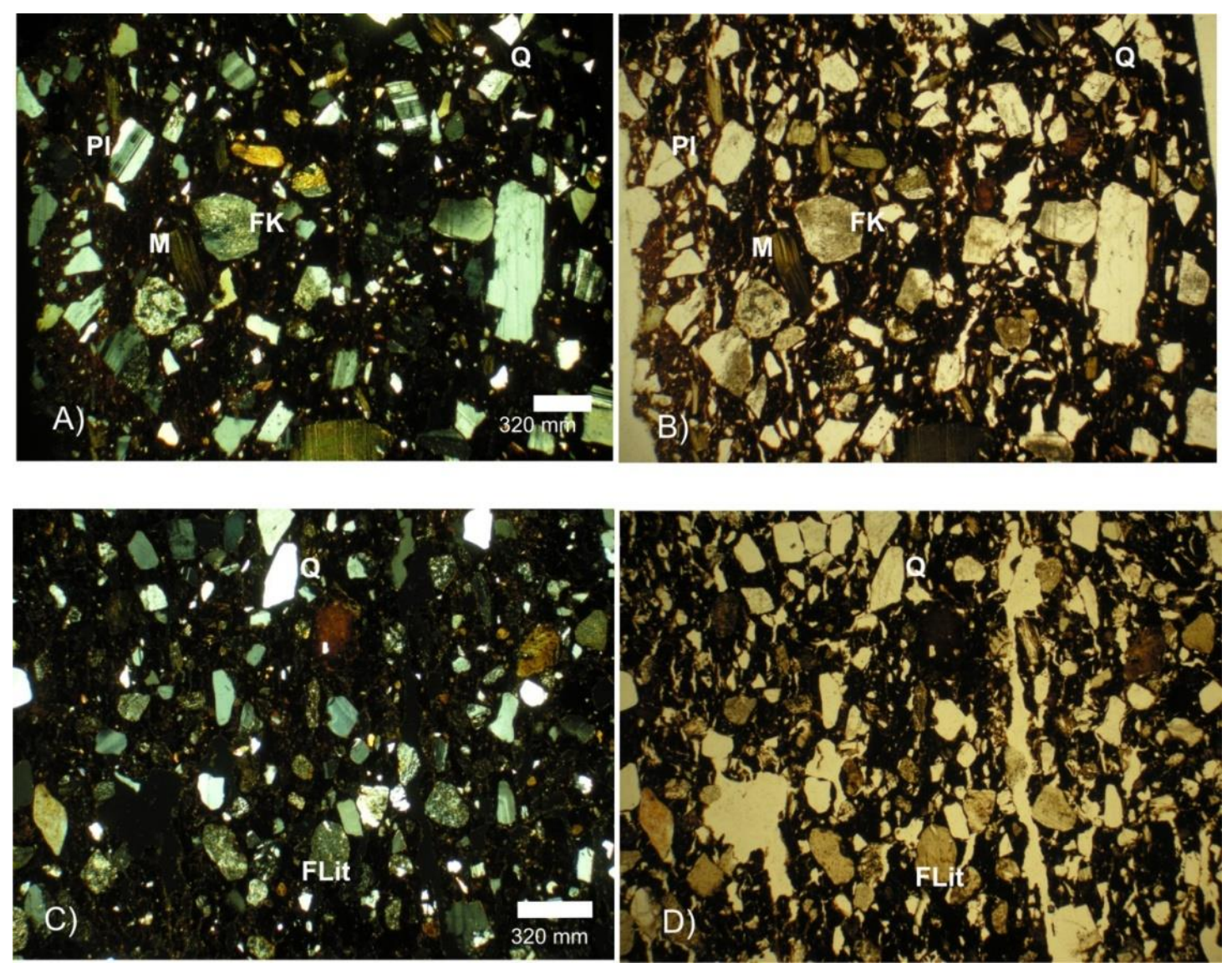

Figura 5. Fotomicrografía de cortes delgados de cerámica de los sitios SP7 y SP9 (Con/sin nicoles). a

y b) muestra124: 10X; c y d) muestra 127: 2.5X. Nota: Pl. (plagioclasa), M (mica), FK (feldespato potásico), FLit. (fragmento lítico), Q (cuarzo)

Finalmente, se observaron algunas generalidades de las pastas: la textura media es la más representada, las inclusiones se distribuyen homogéneamente, los colores pardos son los más representados (Tabla 4) y las cavidades o poros se manifiestan habitualmente en bajo porcentaje (entre $5 \%$ y 10\%), con formas elongadas y distribución irregular. 


\begin{tabular}{|c|c|c|c|c|c|c|c|}
\hline 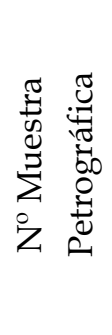 & 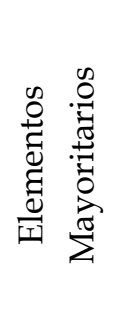 & 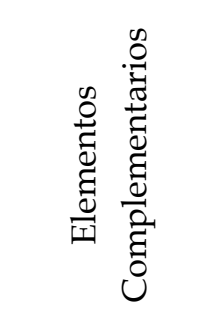 & 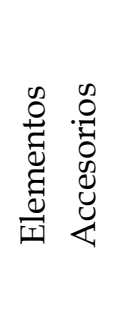 & 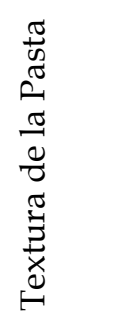 & 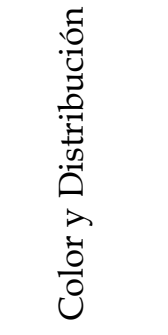 & 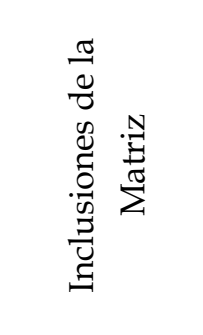 & 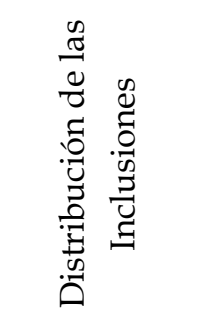 \\
\hline 124 & $\begin{array}{c}\text { F. litalt., } \\
\text { Biot, } \mathrm{Pl}, \\
\text { Q }\end{array}$ & $\begin{array}{l}\text { Feld. pot. alt., } \\
\text { Min. opacos, }\end{array}$ & Anf, Px & Media & $\begin{array}{l}\text { 5YR2/2 } \\
\text { uniforme }\end{array}$ & Mica & Heterogénea \\
\hline 125 & $\begin{array}{c}\text { F. litalt., } \\
\text { Pl, Q }\end{array}$ & $\begin{array}{l}\text { Feld. pot. alt., } \\
\text { Min. opacos, }\end{array}$ & Anf, Px & Media & $\begin{array}{c}\text { 5YR2/2 } \\
\text { No } \\
\text { uniforme }\end{array}$ & $\begin{array}{c}\text { Vidrio } \\
\text { volcánico }\end{array}$ & Heterogéneo \\
\hline 126 & $\begin{array}{c}\text { F. litalt., } \\
\mathrm{Pl}, \mathrm{Q}\end{array}$ & $\begin{array}{l}\text { Feld. pot. alt., } \\
\text { Min. opacos, }\end{array}$ & Anf, Px & $\begin{array}{l}\text { Fina/ } \\
\text { Media }\end{array}$ & $\begin{array}{l}\text { 5YR2/2 } \\
\text { Uniforme }\end{array}$ & $\begin{array}{l}\text { Mica, vidrio } \\
\text { volcánico }\end{array}$ & Homogéneo \\
\hline 127 & $\begin{array}{c}\text { F. litalt., } \\
Q\end{array}$ & $\begin{array}{l}\text { Biot, Feld. } \\
\text { pot. alt. }\end{array}$ & $\begin{array}{l}\text { Min. } \\
\text { opacos }\end{array}$ & Gruesa & $\begin{array}{l}\text { 5YR2/2 } \\
\text { Uniforme }\end{array}$ & Mica & Homogéneo \\
\hline 128 & $\begin{array}{c}\text { F. litalt., } \\
\text { Pl, Q }\end{array}$ & feld. pot. alet. & $\begin{array}{c}\text { Anf, Px } \\
\text { Min. } \\
\text { opacos }\end{array}$ & Media & $\begin{array}{l}\text { 5YR2/2 } \\
\text { Uniforme }\end{array}$ & $\begin{array}{l}\text { Mica, vidrio } \\
\text { volcánico }\end{array}$ & Homogéneo \\
\hline 129 & $\begin{array}{c}\text { F. litalt., } \\
\text { Pl, Q }\end{array}$ & Feld. pot. Alt. & $\begin{array}{c}\text { Anf, Px, } \\
\text { Min. } \\
\text { Opacos }\end{array}$ & Media & $\begin{array}{l}\text { 5YR2/2 } \\
\text { Uniforme }\end{array}$ & $\begin{array}{l}\text { Mica, vidrio } \\
\text { volcánico }\end{array}$ & Homogéneo \\
\hline
\end{tabular}

Tabla 4. Composición mineralógica de los cortes delgados de la localidad San Pablo.Nota: Q (Cuarzo), Feld. (feldespato potásico), Pl. (plagioclasa), anf. (anfibol), Px. (piroxeno), biot (biotita), f.lit.alt. (fragmento lítico alterado), min. opacos (minerales opacos).

\section{Discusión y conclusiones}

Los estudios geológicos y geomorfológicos emprendidos en el área permitieron estimar que hace 3000 años AP el nivel marino se encontraba por encima del actual, y por ello, el ambiente donde se encuentran emplazados los sitios con registro cerámico descriptos para esta localidad arqueológica no habrían estado disponibles para la ocupación humana (Gómez Otero et al. 2017a).

Hasta el momento, los materiales cerámicos han sido registrados en cotas por debajo de los $10 \mathrm{msnm}$. Particularmente en estos sitios, se observa que los conjuntos líticos muestran otros rasgos tecnológicos diferentes respecto de los de las cotas más altas, como por ejemplo, aumento en la proporción de rocas alóctonas, 
aprovechamiento exhaustivo de los nódulos de mejor calidad para la talla (sílices, calcedonias y xilópalos), tamaños más pequeño de los artefactos y reactivación de filos retocados. Esto sugeriría mayor tiempo de permanencia en estos lugares, más inversión de trabajo en la talla, y también, el abastecimiento o circulación de rocas por fuera de la Península (Gómez Otero et al. 2017a).

La localidad San Pablo se destaca por la cantidad de materiales cerámicos registrados, y si bien los parámetros de preservación de los mismos resultan dispares entre las distintas concentraciones cerámicas reconocidas, en general los fragmentos no presentan alteraciones y una importante cantidad de ellos pudo ser ensamblado y reconstruido.

Los ambientes dunarios han sido contantemente utilizados por los cazadores recolectores del área (Gómez Otero 2007), dejando incluso piezas cerámicas completas, posiblemente, como parte del equipamiento de los sitios para sus próximos retornos. Esto que se ha propuesto para algunos sitios de Península Valdés -Las Ollas 1 y La Azucena 2 (Schuster 2014)- también puede ser planteado para la localidad arqueológica San Pablo (particularmente en SP6), ya que si bien muchas de las piezas cerámicas recuperadas no pudieron remontarse completamente, la presencia de fracturas frescas de los tiestos permiten inferir que las piezas se habrían quebrado in situ por factores postdepositacionales, y no habrían sufrido erosión ni transporte. A partir de la ejecución de una práctica tafónomica realizada con materiales cerámicos experimentales, se pudo estimar que la rápida migración de los fragmentos en contextos dunarios es una de las posibles causas por la que no se recobran la totalidad de los fragmentos en las excavaciones.

Retomando el concepto de equipamiento del espacio, cabe mencionar que en los sitios cerámicos del área en los que se ha planteado esta cualidad, también son frecuentes los artefactos de molienda. La cerámica, si bien no tan pesada como los otros artefactos pero si de mayor fragilidad, podría haber estado equipando de manera permanente o semipermanante estos espacios (Schuster 2014). En el sentido de Eerkens (2003) representaría una estrategia alternativa de "almacenamiento de vasijas" para evitar su acarreo y traslado permanente. En tal sentido, este autor plantea que la presencia de cerámica no se encuentra en estrecha vinculación con la movilidad residencial de los grupos sino con la recurrencia en la utilización de determinados lugares.

Respecto de los contextos arqueológicos en los que se registró cerámica, cabe mencionar que en algunos casos este material se presentaba discretamente agrupado junto a restos faunístico o materiales líticos- aunque en otros casos se encontraban dispersos y/o sin ninguna otra evidencia asociada. Asimismo, se indica que no se recuperó material cerámico en entierros humanos, tal como había sido observado anteriormente en el área de estudio (Schuster 2014, 2019). No obstante, se destaca la 
asociación espacial de una pieza cerámica en la misma hoyada de deflación en la que se registraron cinco conjuntos o paquetes óseos de guanacos de características muy particulares, y que hasta el momento, representan un contexto único en la región patagónica.

La cerámica de San Pablo posee características -en cuanto a forma y técnicas de acabado de superficie- que concuerdan con el repertorio cerámico estudiado anteriormente, y que ha sido definido de producción local (Schuster 2009, 2014). El modo de elaboración más común fue la superposición de rollos o rodetes, que posteriormente fueron alisados y /o pulidos. Mediante esta técnica se modelaron ollas de perfiles simples globulares o subglobulares de bases cóncavas- de bordes rectos o levemente evertidos y con diámetros de boca relativamente pequeños (no mayores a los $22 \mathrm{~cm}$ ). Todas estas propiedades tecnológicas habrían favorecido las cocciones prolongadas, sin evaporación de los líquidos, optimizando así el uso de la leña disponible localmente. Respecto de la única pieza completa, la reconstrucción y análisis digital en 3D a la que fue sometida permitieron estimar que es una olla que habría podido contener un poco menos de dos litros (Schuster y Quinto Sánchez 2019). Además, el escaso peso de la misma -tanto vacía como completa- permitió deducir que habría sido muy eficaz para su uso en tareas culinarias y/o para su transporte (Schuster y Quinto Sánchez 2019, Schuster 2019). Esta pieza y otras que han podido ser reconstruidas parcialmente en la localidad San Pablo mantienen las características morfológicas en común con otras ya descriptas para el área de estudio, y por ello también, mantendrían la propiedad multifuncional mencionada anteriormente en la región (Schuster 2014, 2019). Esta propiedad o cualidad tiene fundamento en las formas generalizadas de las piezas que fueron útiles para cubrir una amplia variedad de usos, como por ejemplo, preparar, cocinar o almacenar alimentos, y en menor medida, líquidos (Schuster 2014). Además, este tipo de diseños versátiles habrían sido funcionales al modo de vida cazador recolector móvil de estas poblaciones.

De acuerdo con los estudios microscópicos, la mayor parte de la cerámica analizada fue elaborada con materiales arcillosos disponibles localmente. Para otros sitios arqueológicos de Península Valdés y de la costa nordeste del Chubut también se ha propuesto el uso de materias primas locales para la confección de la amplia mayoría de las cerámicas registradas (Schuster 2009, 2014, 2015, 2019). Con los datos recabados en esta nueva localidad arqueológica, se mantiene la hipótesis de que las escasas piezas de procedencia alóctona son las que presentan mica como componente mayoritario en las pastas (Schuster 2014, 2019), y que generalmente además, presentan morfologías o rasgos particulares como bordes reforzados, cuellos y/o asas. El caso aquí presentado -con asas en cinta- se correspondería con la única cerámica alóctona reconocida hasta el momento en la localidad San Pablo, y singularmente, fue registrada en un contexto específico y particular como son los paquetes de restos óseos de guanaco ya mencionados. 
Por otra parte, en las paredes externas de un amplio grupo de fragmentos se observaron manchas de hollín que estarían sugiriendo que los contenedores cerámicos se apoyaban directamente sobre el fuego o sobre las brasas durante la cocción de los alimentos. No obstante, hay que tener en cuenta que parte del material cerámico fue recuperado en un contexto de basural junto a otros ítems arqueológicos con señales de combustión. Además, se registraron restos orgánicos adheridos en algunas caras internas de los tiestos, materiales que están siendo estudiados actualmente mediante cromatografía gaseosa para determinar cuáles fueron los productos -vegetales y/o animales- que se preparaban, cocinaban y/o almacenaban en estos contenedores. Hasta el momento, los resultados alcanzados en la región, están indicando el procesamiento de variados alimentos terrestres y marinos -vegetales, guanaco, pinnípedos y peces- como parte de un aprovechamiento integral de los recursos disponibles (Gómez Otero et al. 2014a y b; Schuster 2014). Particularmente de interés son los resultados que se obtengan de la única pieza completa que presentaba una cobertura orgánica macroscópica en la su circunferencia interna, y que corresponde seguramente con la y/o las últimas comidas procesadas en la misma. Otro caso que reviste importancia, es la pieza con asas asociada a los paquetes de guanacos. Al ser una olla alóctona, es posible que los resultados de residuos orgánicos se muestren completamente disímiles a los ya obtenidos en la región. Esta línea de evidencia, así como las tareas de experimentación tafonómica de tiestos llevada a cabo con la finalidad de establecer parámetros que ayuden a interpretar el material cerámico recolectado en las tareas arqueológicas- permitirán el desarrollo y la continuidad de las investigaciones cerámicas en esta localidad arqueológica en particular y en la región costera del noreste del Chubut, así como también favorecerán las interpretaciones acerca de la implementación y uso de esta tecnología a nivel regional.

Agradecimientos: Al Sr. Juan Ignacio Mihalich, quien facilitó para su estudio la pieza completa; al Sr. Manolo Arias y los guardaparques Esteban Bremer, Rafael Lorenzo y Carlos Saibene de la Fundación Vida Silvestre por la asistencia en los trabajos de campo; a mi sobrina Abril Monticelli por su grata compañía y colaboración en algunas de las tareas emprendidas juntas en el campo y en el laboratorio.

\section{Bibliografía Citada}

Adán L. A., R. Mera, M. Uribe y M. Alvarado

2005 La tradición cerámica bícroma rojo sobre blanco en la región sur de Chile: los estilos decorativos Valdivia-Vergel. Actas XVI Congreso Nacional de Arqueología Chilena: 399-410. Museo de Historia Natural. Concepción, Chile. 
Beeskow, A. M.

2002 Usos tradicionales de las plantas en la meseta patagónica. Jardín Botánico de la Patagonia Extraandina Centro Nacional Patagónico (CENPAT-CONICET) - International Cooperative Biodiversity Group (ICBG). Puerto Madryn, Chubut.

Beeskow, A. M, H. F. Del Valle y C. M. Rostagno

1987 Los sistemas fisiográficos de la región árida y semiárida de la provincia de Chubut. CENPATCONICET, SECyT y Delegación Regional Patagonia. Puerto Madryn, Chubut.

Bullock D. S.

1973 La cultura Kofkeche. Boletín de la Sociedad de Biología de Concepción, XLIII: 1-207

Cabrera A. L.

1976 Regiones fitogeográficas Argentinas. Enciclopedia Argentina de Agricultura y Ganadería. Ed. Acme. Buenos Aires.

Coronato, F.

1994 Clima del nordeste del Chubut. Séptima Reunión de Campo. CADINQUA. Guía de Campo Península Valdés y Centro Noroeste del Chubut: 13-20. Centro Nacional Patagónico (CENPAT-CONICET). Puerto Madryn, Chubut.

Escofet A. M., J. M. Orensanz, S. R. Olivier y V. Scarabino 1978 Biocenología bentónica del golfo San Matías (Río Negro): metodología, experiencias y resultados del estudio ecológico de un gran espacio geográfico de America Latina. Anales del Centro Científico del mar y limnológico 5 (1): 59-82.

Garrido L. J., J. N. Amaya y Z. Kovackz

1981 Territorialidad, comportamiento individual y actividad diaria de una población de guanacos en la reserva faunística de Cabo Dos Bahías. Contribución 42. Centro Nacional Patagónico (CENPAT-CONICET). Puerto Madryn, Chubut.

Gómez Otero, J.

2007 Recursos, dieta y movilidad en la costa centro-septentrional de Patagonia durante el Holoceno medio y tardío. Tesis doctoral inédita. Facultad de Filosofía y Letras, UBA. Buenos Aires.

Gómez Otero J., Banegas A., Caruso Femme L., M.S. Goye, V. Schuster, A. Sbodova y N. Weiler.

2017a Los primeros pobladores humanos: arqueología de la Bajada Colombo. San Pablo de Valdés. 10 años. Ed. Fundación Vida Silvestre, Buenos Aires. 
Gómez Otero J, Schuster V. y A. Banegas

2017b Archaeology of the Península Valdés: spatial and temporal variability in the human use of the landscape and geological resources. Late Cenozoic of Penísula Valdés: an interdisciplinary approach. Chapter 10, pp. 233-261. Ed. SPRINGER, Canadá.

Gómez Otero J, V. Schuster y A. Svodoba.

2014a Fish and plants: the hidden resources in the archaeological record of the northcentral Patagonian coast (Argentina). Quaternary International XXX: 1-10.

Gómez Otero J., V. Schuster y D. Constenla

2014b Isótopos estables de carbono y nitrógeno y cromatografía gaseosa en cerámica del nordeste de la Provincia del Chubut (Patagonia Argentina). Arqueología 20 (2): 263-284.

Gómez Otero J., A. Banegas, M.S. Goye, D. Palleres, M. Reyes, V. Schuster y A. Svoboda 2013 Nuevas investigaciones arqueológicas en la Estancia San Pablo (costa del Golfo Nuevo, Península Valdés). En Arqueología Argentina en el Bicentenario de la Asamblea General Constituyente del año 1813, J. R. Bárcena y S. E. Martín Editores, pp. 523-524. Universidad Nacional de la Rioja, INCIHUSA-CONICET, La Rioja.

Gómez Otero J., J. B. Belardi, A. Súnico y R. Taylor 1999 Arqueología de cazadores-recolectores en Península Valdés, costa central de Patagonia: primeros resultados. En Soplando en el viento (Actas de las III Jornadas de Arqueología de la Patagonia), pp. 393-417. INAPL - Universidad Nacional del Comahue, Neuquén- Buenos Aires.

Haller, M. J.

1994 Características geológicas regionales. Séptima Reunión de Campo. CADINQUA. Guía de

Campo Península Valdés y Centro Noroeste del Chubut: 12-13. Centro Nacional Patagónico (CENPAT-CONICET). Puerto Madryn, Chubut.

Haller, M. J., C. M. Meister, A. J. A. Monti y N. Weiler

2005 Descripción de la hoja geológica 4366-II, Puerto Madryn, provincia del Chubut. Programa Nacional de Cartas Geológicas 1:250.000. Boletín 289. Servicio Geológico Minero Argentino. Buenos Aires.

Haller, M. J., A. J. A. Monti y C. M. Meister 2001 Descripción de la hoja geológica 4363-I, Península Valdés, provincia del Chubut. Boletín 266. Servicio Geológico Nacional. Buenos Aires. 
Munita D., L. Adán y C. R. Mera

2011 Prospecciones arqueológicas terrestres en áreas lacustres, piemontana, cordillerana y pampeana del centro sur de Chileno. Magallania 38 (1): 247-268.

Orton C, P. Tyers y A. Vince

1997 La Cerámica en Arqueología. Ed. Crítica. Barcelona.

Scasso, R. A. y C. Del Río

1987 Ambiente de sedimentación, estratigrafía y proveniencia de la secuencia marina del Terciario Superior de la región de Península Valdés, Chubut. Asociación Geológica Argentina XLII (3-4): 291-321.

Schuster, V.

2009 Petrografía de la cerámica arqueológica del nordeste del Chubut (Patagonia Argentina). Primeros resultados. En S. Bertolino, R. Cattanéo y A. D. Izeta (Eds.), La Arqueometría en Argentina y Latinoamérica, pp. 103-108. Facultad de Filosofía y Humanidades Ediciones, Córdoba.

2014 La organización tecnológica de la cerámica de cazadores-recolectores. Costa norte de la Provincia del Chubut (Patagonia Argentina). Relaciones XXXIX (1): 203-231.

2015 Cerámica arqueológica de la costa, valle y meseta de la provincia del Chubut (Patagonia Argentina): estudio comparativo preliminar de la composición de las pastas a través de la petrografía. Intersecciones en Antropología 16 (2): 353-366.

2019 El registro cerámico de cazadores recolectores costeros, provincias de Río Negro y Chubut, Patagonia Argentina. En: V. Schuster y A. E. Pérez (Eds.), Cerámica Arqueológica de la Patagonia. Ed. Fundación F. Azara, Buenos Aires. En Prensa.

Schuster, V. y M. Quinto Sánchez

2019. Reconstrucciones virtuales en 3D del repertorio cerámico de cazadores-recolectores de la costa nordeste del Chubut, Patagonia Argentina. Arqueología 25 (1): 103-114.

Súnico C. A.

1996 Geología del cuaternario y ciencia del suelo: relaciones geomórficas y estratigráficas con suelos y paleosuelos. Tesis Doctoral inédita. Facultad de Ciencias Exactas y Naturales, UBA. Buenos Aires.

Svodoba, A. 2015 Los vertebrados pequeños en la subsistencia de los cazadores-recolectores: una evaluación zooarqueológica comparativa para Patagonia central. Tesis doctoral inédita, Facultad de Filosofía y Letras, UBA. Bueno Aires 
Vázquez, N. N.

2004 Biodiversidad costero marina en la Patagonia: características, conservación e importancia. Fundación Patagonia Natural y Wild life Conservation Society. Puerto Madryn, Chubut. 